\title{
CARACTERIZAÇÃO DE PACIENTES COM TRANSTORNOS MENTAIS DE UM HOSPITAL GERAL E DE ENSINO*
}

\author{
Marcio Roberto Paes¹, Mariluci Alves Maftum², Jorge Vinícius Cestari Felix³, \\ Maria de Fatima Mantovani ${ }^{4}$, Thais Aidar de Freitas Mathias ${ }^{5}$
}

\begin{abstract}
RESUMO: Objetivo: caracterizar o perfil sociodemográfico e clínico dos pacientes com transtornos mentais em hospital geral. Método: estudo quantitativo, transversal, com 179 pacientes com diagnósticos de transtornos mentais. Os dados foram coletados entre 2012 e 2013 em unidades de internação de um hospital geral por meio de instrumentoestruturado, analisados por métodos estatístico-descritivos e, posteriormente apresentados em frequências. Resultados: as mulheres apresentaram maior prevalência de transtornos do humor e os homens de transtornos relacionados ao uso de substâncias psicoativas. A unidade de neurologia teve o maior percentual dos casos (27,4\%); 67\% faziam tratamento psiquiátrico; $77,7 \%$ usavam medicações psicotrópicas contínuas; 23,5\% referiram tentativa de suicídio; 21,2\% apresentavam comorbidadespsiquiátricas; 25,3\% eram dependentes de álcool, 30,7\% de tabaco e 17,3\% de outras drogas. Conclusão: as particularidades apresentadas pelos participantes subsidiam o reconhecimento de sinais e sintomas dos transtornos mentais e oplanejamento de cuidados de enfermagem específicos a esta clientela em hospital geral. DESCRITORES: Perfil de Saúde; Saúde Mental; Enfermagem; Enfermagem Psiquiátrica; Hospitais Gerais. ES: Saúde do trabalhador; Notificação; Acidentes de trabalho; Doenças ocupacionais; Sistema de Informação em Saúde.
\end{abstract}

\section{CHARACTERIZATION OF PATIENTS WITH MENTAL DISORDERS FROM A GENERAL TEACHING HOSPITAL*}

ABSTRACT: Objective: to characterize the sociodemographic and clinical profile of patients with mental disorders in a general hospital. Method: a quantitative, cross-sectional study with 179 patients with diagnoses of mental disorders. The data were collected between 2012 and 2013 in hospitalization units of a general hospital by means of a structured instrument, analyzed by statistical-descriptive methods and later presented as frequencies. Results: the women presented higher prevalence of mood disorders and the men of psychoactive substance use disorders. The neurology unit had the highest percentage of cases $(27.4 \%) ; 67 \%$ were receiving psychiatric treatment; $77.7 \%$ used continuous psychotropic medication; $23.5 \%$ reported suicide attempts; $21.2 \%$ had psychiatric comorbidities; $25.3 \%$ were dependent on alcohol, $30.7 \%$ on tobacco and $17.3 \%$ on other drugs. Conclusion: the particularities presented by the participants will help in the recognition of signs and symptoms of mental disorders and the planning of nursing care specific to this clientele in a general hospital.

KEYWORDS: Health Profile; Mental Health; Nursing; Psychiatric Nursing; General Hospitals.

\section{CARACTERIZACIÓN DE PACIENTES CON TRASTORNOS MENTALES EN UN HOSPITAL GENERAL E DE ENSEÑANZA*}

RESUMEN: Objetivo: caracterizar el perfil social demográfico y clínico de los pacientes con trastornos mentales en hospital general. Método: estudio cuantitativo, transversal, con 179 pacientes con diagnósticos de trastornos mentales. Se recogieron los datos entre 2012 y 2013 en unidades de internación de un hospital general por medio de instrumento estructurado y se los analizaron por métodos estadísticos descriptivos, siendo posteriormente presentados en frecuencias. Resultados: las mujeres presentaron más prevalencia de trastornos de humor y los hombres, de trastornos asociados al uso de sustancias psicoactivas. La unidad de neurología tuvo el mayor porcentual de los casos (27,4\%); $67 \%$ hacían tratamiento psiquiátrico; $77,7 \%$ usaban medicaciones psicotrópicas continuas; $23,5 \%$ relataron tentativa de suicidio; $21,2 \%$ presentaban comorbilidades psiquiátricas; $25,3 \%$ eran dependientes de alcohol, 30,7\% de tabaco y 17,3\% de otras drogas. Conclusión: las particularidades presentadas por los participantes subsidian el reconocimiento de señales y síntomas de los trastornos mentales y el planeamiento de cuidados de enfermería específicos a ese tipo de paciente en hospital general.

DESCRIPTORES: Perfil de Salud; Salud Mental; Enfermería; Enfermería Psiquiátrica; Hospitales Generales.

\footnotetext{
*Artigo extraído da Tese de Doutorado intitulada: "Perfil e diagnósticos de enfermagem de pacientes com transtornos mentais em um hospital geral e de ensino", Programa de Pós-Graduação em Enfermagem Universidade Federal do Paraná, Curitiba, PR, Brasil, 2013.

${ }^{1}$ Enfermeiro. Doutor em Enfermagem. Docente do Departamento de Enfermagem da Universidade Federal do Paraná. Curitiba, PR, Brasil.

${ }^{2}$ Enfermeira. Doutora em Enfermagem. Docente do Departamento de Enfermagem da Universidade Federal do Paraná. Curitiba, PR, Brasil.

${ }^{3}$ Enfermeiro. Doutor em Fisiologia Humana. Docente do Departamento de Enfermagem da Universidade Federal do Paraná. Curitiba, PR, Brasil.

${ }^{4}$ Enfermeira. Doutora em Enfermagem. Docente do Departamento de Enfermagem da Universidade Federal do Paraná. Curitiba, PR, Brasil.

${ }^{5}$ Enfermeira. Doutora em Saúde Publica - Epidemiologia. Docente do Departamento de Enfermagem da Universidade Estadual de Maringá. Maringá, PR, Brasil.
} 


\section{INTRODUÇÃO}

Nas últimas décadas, as questões relacionadas com a saúde mental da população têm sido priorizadas na atenção à saúde em todo o mundo. Isso porqueháprevalência global dos transtornos mentais e dos problemas psicossociais causados pelo uso do álcool e de outras drogas, configurando-se em grave questão de saúde pública e, consequentemente, foco de discussões e pesquisas da comunidade científica. (1)

Estima-se que 25\% da população mundial (um em cada quatro indivíduos) apresentem,pelo menos, um transtorno mental em dada fase de sua vida. Os últimos dados disponibilizados pela Organização Mundial de Saúde indicam que aproximadamente 450 milhões de pessoas no mundo sofram de transtornos mentais ou de transtornos relacionados ao uso nocivo de álcool e outras drogas. ${ }^{(2)}$

Esses números refletidos em serviços de saúde, principalmente em hospitais gerais, dão conta que em torno de $30 \%$ dos pacientes internados para tratamento clínico ou cirurgico fazem uso de substâncias psicoativas (álcool e outras drogas) e a maioria desses pacientes apresenta doenças clínicas relacionadas com a dependência química ou um transtorno mental. ${ }^{(3-4)}$

A assistência a estes pacientes em hospitais gerais se torna mais complexa em relação aos outros, por suas particularidades e pelo comportamento da equipe de saúde que os atende. Isto porque, muitas vezes, tais equipes não estãopreparadas para suprir as necessidades de assistência ao quadro clínico conjuntamente às condições psicossociais. ${ }^{(3,5-7)}$

Resultados de estudos demonstram que os profissionais de saúde de hospitais gerais apresentam dificuldades de cuidar adequadamente desses pacientes. Usualmente, os pacientes internados em hospitais gerais têm o seu tratamento focado nas alterações físico-biológicas e, em grande parte, as dimensões psicossociais não são contempladas nos tratamentos pelos profissionais de saúde. ${ }^{(3,5-7)}$

Atualmente, reconhece-sea existência de déficit na formação, no conhecimento e na habilidade dos profissionais de enfermagemno desenvolvimento de cuidados aos pacientes com transtornos mentais em hospitais gerais. Contudo, esta realidade precisa ser transformada emergencialmente, visto que as políticas públicas de saúde mental no Brasil têm considerado, enfaticamente, o hospital geral imprescindível na rede de atenção psicossocial. ${ }^{(8)}$

Assim, acredita-se que o conhecimento de uma população com maior risco de sofrer de um agravo específico, contribui para o planejamento e a implementação de políticas nacionais, regionais e locais, e subsidia profissionais da área da saúde na escolha das melhores práticas assistenciais a serem ofertadas a tais pessoas. Assim, a compreensão de como as questões relacionadas com a saúde mental se comportam, contribui para o avanço das ações de promoção, prevenção e tratamento das pesssoas acometidas por transtornos mentais.

A questão problema para esta pesquisa foi: Qual o perfil sociodemográfico e clínico de pacientes com transtornos mentais internados em um hospital geral? Para responder a esta questão, propôs-se o seguinte objetivo:caracterizar o perfil sociodemográfico e clínico dos pacientes com transtornos mentais.

\section{MÉTODO}

Trata-se de estudo quantitativo, transversal,desenvolvido em seis unidades de internamento de um hospital geral e de ensino: Clínica Médica Masculina (CMM); Clínica Médica Feminina (CMF), Neurologia, Cardiologia, Infectologia e Pronto-atendimento (PA).

Participaram deste estudo 179 pacientes com transtornos mentais,selecionados pelo procedimento de amostragem não-probabilística e por conveniência. Os critérios de inclusão foram: possuir diagnóstico 
médico detranstorno mental descrito em prontuário e ter 18 anos ou mais. Os critérios de exclusão foram: pacientes já entrevistados, reinternamentos em uma das unidades do local de estudo,e estado de coma ou rebaixamento de nível de consciência.

A coleta de dados ocorreu no período de fevereiro de 2012 a janeiro de 2013 por meio de um instrumento estruturado, em que parte dos dados foi obtida a partir de informações descritas nos prontuários: idade, sexo, procedência, instrução, ocupação, religião, unidade de internamento, doença clínica principal, transtorno mental, uso de medicação psiquiátrica. As demais foram perguntadas aos participantes: cor da pele (autorreferida), com quem reside, situação conjugal, ocupação, renda familiar,tratamento psiquiátrico, histórico de uso drogas e de comportamento suicida.

Os dados foram armazenados em planilha criada pelo SoftwareStatistical Package for the Social Sciences SPSS ${ }^{\circledR}$ 13.0, com dupla digitação independente. Após a verificação e correção de eventuais erros de digitação e inconsistências, os dados foram analisados por métodos descritivos e apresentados sob a forma de tabela e gráficos.Os resultados obtidos pela análise descritiva estão expressos por medidas de tendência central para variáveis numéricas, e as variáveis categóricas estão apresentadas por frequência e percentuais.

O Projeto de Pesquisa foi aprovado pelo Comitê de Ética em Pesquisa do Hospital de Clínicas da Universidade Federal do Paraná (UFPR), parecer no 2577.184/2011-08.

\section{RESULTADOS}

\section{Perfil sociodemográfico e clínico}

Durante o período de coleta dos dados houve 2.532 internamentosde pacientes nas unidades em que o estudo foi realizado. Considerando os 179 casos, a prevalência de pacientes com transtornos mentaisfoi de 7,1\%, dos quais $96(53,6 \%)$ eram do sexo feminino. A média de idade da amostra foi de 48,95 anos. Em relação às variáveis sociais, observou-seque houve prevalência de participantes casados, religião católica, ensino fundamental incompleto, homens prestadores de serviço ou mulheres sem profissão, aposentados, com renda familiar entre dois e quatro salários mínimos, conforme apresentado na Tabela 1.

Tabela 1 - Distribuição dos participantes, segundo as variáveis sociais. Curitiba, PR, Brasil, 2013. (continua)

\begin{tabular}{|c|c|c|c|c|c|c|}
\hline \multirow{3}{*}{ Variáveis sociais } & \multicolumn{4}{|c|}{ Sexo } & \multirow{2}{*}{\multicolumn{2}{|c|}{ Total }} \\
\hline & \multicolumn{2}{|c|}{ Masculino } & \multicolumn{2}{|c|}{ Feminino } & & \\
\hline & (n) & $\%$ & (n) & $\%$ & (n) & $\%$ \\
\hline \multicolumn{7}{|l|}{ Situação conjugal } \\
\hline Casado/relação estável & 44 & 24,6 & 41 & 22,9 & 85 & 47,5 \\
\hline Solteiro & 27 & 15,1 & 23 & 12,8 & 50 & 27,9 \\
\hline Divorciado & 10 & 5,6 & 15 & 8,4 & 25 & 14 \\
\hline Viúvo & 2 & 1,1 & 17 & 9,5 & 19 & 10,6 \\
\hline \multicolumn{7}{|l|}{ Religião } \\
\hline Católica & 56 & 31,3 & 55 & 30,7 & 111 & 62 \\
\hline Protestante/evangélica & 11 & 6,1 & 34 & 19 & 45 & 25,1 \\
\hline Espírita & 1 & 0,6 & 3 & 1,7 & 4 & 2,2 \\
\hline Afro-brasileira & 1 & 0,6 & --- & --- & 1 & 0,6 \\
\hline Budista/oriental & 1 & 0,6 & --- & --- & 1 & 0,6 \\
\hline
\end{tabular}




\begin{tabular}{|c|c|c|c|c|c|c|}
\hline Outras & 3 & 1,7 & 3 & 1,7 & 6 & 3,4 \\
\hline Sem religião & 10 & 5,6 & 1 & 0,6 & 11 & 6,1 \\
\hline \multicolumn{7}{|l|}{ Instrução } \\
\hline Analfabeto & 2 & 1,1 & 5 & 2,8 & 7 & 3,9 \\
\hline Ensino fundamental incompleto & 37 & 20,7 & 52 & 29,1 & 89 & 49,8 \\
\hline Ensino fundamental completo & 11 & 6,1 & 10 & 5,6 & 21 & 11,7 \\
\hline Ensino médio incompleto & 6 & 3,4 & 6 & 3,4 & 12 & 6,7 \\
\hline Ensino médio completo & 19 & 10,6 & 13 & 7,3 & 32 & 17,9 \\
\hline Ensino superior incompleto & 3 & 1,7 & 4 & 2,2 & 7 & 3,9 \\
\hline Ensino superior completo & 5 & 2,8 & 4 & 2,2 & 9 & 5 \\
\hline Pós-graduação & --- & --- & 2 & 1,1 & 2 & 1,1 \\
\hline \multicolumn{7}{|l|}{ Profissão (área de atuação) } \\
\hline Saúde & --- & --- & 6 & 3,4 & 6 & 3,4 \\
\hline Educação & 1 & 0,6 & 3 & 1,7 & 4 & 2,2 \\
\hline Construção civil & 12 & 6,7 & --- & --- & 12 & 6,7 \\
\hline Administração & 1 & 0,6 & 3 & 1,7 & 4 & 2,2 \\
\hline Comércio & 10 & 5,6 & 6 & 3,4 & 16 & 8,9 \\
\hline Indústria & 2 & 1,1 & --- & --- & 2 & 1,1 \\
\hline Prestação de serviços & 43 & 24 & 26 & 14,5 & 69 & 38,5 \\
\hline Trabalho rural & 2 & 1,1 & 2 & 1,1 & 4 & 2,2 \\
\hline Sem profissão & 12 & 6,7 & 50 & 27,9 & 62 & 34,6 \\
\hline \multicolumn{7}{|l|}{ Ocupação } \\
\hline Do lar & --- & --- & 30 & 16,8 & 30 & 16,8 \\
\hline Estudante & 2 & 1,1 & 3 & 1,7 & 5 & 2,8 \\
\hline Desempregado & 6 & 3,4 & 3 & 1,7 & 9 & 5 \\
\hline Empregado assalariado & 22 & 12,3 & 17 & 9,5 & 39 & 21,8 \\
\hline Autônomo & 25 & 14 & 9 & 5 & 34 & 19 \\
\hline Aposentado & 24 & 13,4 & 22 & 12,3 & 46 & 25,7 \\
\hline Sem ocupação & 4 & 2,2 & 12 & 6,7 & 16 & 8,9 \\
\hline \multicolumn{7}{|l|}{ Renda familiar } \\
\hline 01 salário mínimot & 14 & 7,8 & 12 & 6,7 & 26 & 14,5 \\
\hline 02-04 salários mínimos & 55 & 30,7 & 70 & 39,1 & 125 & 69,8 \\
\hline 05 ou mais salários mínimos & 11 & 6,1 & 9 & 5 & 20 & 11,2 \\
\hline Não souberam informar & 3 & 1,7 & 5 & 2,8 & 8 & 4,5 \\
\hline
\end{tabular}

†Considerou-se o salário mínimo no valor de R\$ 622,00, referente ao ano-base 2012

A Tabela 2 apresenta os resultados referentes às variáveis demográficas com destaque à prevalência de participantes com cor de pele autorreferida branca, naturais do interior do estado do Paraná e com procedência de Curitiba ou região metropolitana, moradores da área urbana. 
Tabela 2- Distribuição dos participantes, segundo as variáveis demográficas. Curitiba, PR, Brasil, 2013

\begin{tabular}{|c|c|c|c|c|c|c|}
\hline \multirow{3}{*}{ Variáveis Demográficas } & \multicolumn{4}{|c|}{ Sexo } & \multirow{2}{*}{\multicolumn{2}{|c|}{ Total }} \\
\hline & \multicolumn{2}{|c|}{ Masculino } & \multicolumn{2}{|c|}{ Feminino } & & \\
\hline & $(\mathbf{n})$ & $\%$ & $(\mathbf{n})$ & $\%$ & (n) & $\%$ \\
\hline \multicolumn{7}{|l|}{ Etnia } \\
\hline Branca & 65 & 36,3 & 78 & 43,6 & 143 & 79,9 \\
\hline Preta & 4 & 2,2 & 10 & 5,6 & 14 & 7,8 \\
\hline Parda & 14 & 7,8 & 8 & 4,5 & 22 & 12,3 \\
\hline \multicolumn{7}{|l|}{ Naturalidade } \\
\hline Curitiba & 32 & 17,9 & 24 & 13,4 & 56 & 31,3 \\
\hline Região metropolitana & 5 & 2,8 & 4 & 2,2 & 9 & 5 \\
\hline Interior do Paraná & 28 & 15,6 & 45 & 25,1 & 73 & 40,8 \\
\hline Outro Estado & 17 & 9,5 & 23 & 12,8 & 40 & 22,3 \\
\hline Outro País & 1 & 0,6 & --- & --- & 1 & 0,6 \\
\hline \multicolumn{7}{|l|}{ Procedência } \\
\hline Curitiba & 51 & 28,5 & 51 & 28,5 & 102 & 57 \\
\hline Região metropolitana & 18 & 10,1 & 29 & 16,2 & 47 & 26,3 \\
\hline Interior do Paraná & 9 & 5 & 14 & 7,8 & 23 & 12,8 \\
\hline Outro Estado & 5 & 2,8 & 2 & 1,1 & 7 & 3,9 \\
\hline \multicolumn{7}{|l|}{ Modo de moradia } \\
\hline Mora com familiares & 70 & 39,1 & 88 & 49,2 & 158 & 88,3 \\
\hline Mora sozinho & 12 & 6,7 & 7 & 3,9 & 19 & 10,6 \\
\hline Institucionalizado & 1 & 0,6 & 1 & 0,6 & 2 & 1,1 \\
\hline \multicolumn{7}{|l|}{ Area de moradia } \\
\hline Rural & 4 & 2,2 & 6 & 3,4 & 10 & 5,6 \\
\hline Urbana & 79 & 44,1 & 90 & 50,3 & 169 & 94,4 \\
\hline
\end{tabular}

Referente ao perfil clínico dos participantes, a prevalência dos casos em relação à unidade de internação em que se encontravam no momento da coleta, tem-se que: neurologia, $49(27,4 \%)$ pacientes; CMF, 36(20,1\%); cardiologia, 32(17,9\%); PA, 22(12,3\%); CMM, 22(12,3\%); infectologia,18(10,1\%).

A Tabela 3 apresenta os resultados referentes aos transtornos mentais ou de comportamento descritos nos prontuários dos participantes. Destaque para a prevalência dos transtornos depressivos n=72 (40,2\%).

Tabela 3 - Distribuição dos participantes segundosexo e diagnóstico. Curitiba, PR, Brasil, 2013

\begin{tabular}{lcccccc}
\hline \multirow{2}{*}{ Diagnósticos } & \multicolumn{2}{c}{ Masculino } & \multicolumn{2}{c}{ Feminino } & \multicolumn{2}{c}{ Total } \\
\cline { 2 - 7 } & $(\mathrm{n})$ & $\%$ & $(\mathrm{n})$ & $\%$ & $(\mathrm{n})$ & $\%$ \\
\hline Transtornos depressivos & 19 & 10,6 & 53 & 29,6 & 72 & 40,2 \\
\hline TMCDUłsubstâncias psicoativas & 25 & 14 & 3 & 1,7 & 28 & 15,6 \\
\hline Transtorno afetivo bipolar & 5 & 2,8 & 15 & 8,4 & 20 & 11,2 \\
\hline Transtornos de ansiedade & 5 & 2,8 & 15 & 8,4 & 20 & 11,2 \\
\hline TMCDUł álcool & 18 & 10,1 & 1 & 0,6 & 19 & 10,6 \\
\hline Esquizofrenia & 10 & 5,6 & 4 & 2,2 & 14 & 7,8 \\
\hline Transtornos da alimentação & --- & --- & 3 & 1,7 & 3 & 1,7 \\
\hline Transtornos de personalidade & 1 & 0,6 & 1 & 0,6 & 2 & 1,1 \\
\hline Transtorno somatoforme & --- & --- & 1 & 0,6 & 1 & 0,6 \\
\hline Total & 83 & 46,4 & 96 & 53,6 & 179 & 100 \\
\hline
\end{tabular}

‡TMCDU - Transtorno mental e comportamental devido o uso de. 
Quanto ao perfil clínico, o estudo constatou que 139(77,7\%) faziam uso contínuo de medicação psicotrópica com média de 6,2 anos de uso dessas medicações; 69,5\% com uso de mais de uma classe de medicação psicotrópica. Em relação ao local de tratamento $67 \%$ faziam tratamento psiquiátrico dos quais 53(44,2\%) em ambulatório de saúde mental, 39(32,5\%) em unidades básicas de saúde, 16(13,3\%) em hospitais gerais, 8(6,7\%) em hospitais psiquiátricos, e 4(3,3\%) em Centros de Atenção Psicossocial.

Em relação às comorbidades psiquiátricas 40(22,3\%) dos pacientes apresentaram mais de um diagnóstico psiquiátrico, 90(50,3\%) já haviam pensado em suicídio e 42(23,5\%) já tentaramsuicídio. Foram 44(24,6\%) participantes que afirmaram uso na vidade alguma droga ilícita; desses 37(20,7\%), maconha; 33(18,4\%), cocaína; 25(14\%),crack;e34(19,0\%),outras drogas. Os que referiram uso atual e rotineiroforam 31(17,3\%), dos quais:crack,19(10,6\%); maconha, 17(9,5\%); e cocaína,10(5,6\%).

\section{DISCUSSÃO}

Háprevalência de pacientes com transtornos mentais no hospitalgeral de 7,1\%, menor da encontrada em outros estudos, que variaramentre $30 \%$ e $36,7 \%$ em hospitais brasileiros e colombianos. ${ }^{(9-11)}$ Tais estudos apresentam resultados mais precisos, uma vez que foram desenvolvidos embasados em avaliações de médicos psiquiatras, que realizaram o diagnósticodos pacientes e identificavam a presença de transtornos mentaisnos pacientes de hospitais gerais.

A amostra deste estudo foiconstituída a partir de diagnósticos com base em registro em prontuário, com provável diminuição do real índice de pacientes com transtornos mentais. Isto porque os profissionais de saúde em hospitais gerais têm maior dificuldade de compreender e reconhecer sinais e sintomas de transtornos mentais. Também pode dever-se à crença ainda mantida de que o hospital geral não é o local para atendimento dessa clientela. ${ }^{(7)}$ Isto se confirma ao remeter-se aum estudo similar em hospital geral, a partir de fontes secundárias em que se observoua prevalência de pacientes com transtornos mentais de $2,84 \%$. $^{(12)}$

A pouca diferença percentual entre homens e mulheres, apresentada nos resultados, coaduna com outros estudos. $^{(9,11,13)}$ Contudo, quando se considera alguns transtornos mentais específicos como, por exemplo, transtornos depressivos ou dependência química, observou-sediferença significativa entre homens e mulheres. Os transtornos depressivos e de ansiedade são marcadamente mais prevalentes em mulheres, aproximadamente o dobro em relação aos homens.Todavia, os casos de transtornos relacionados ao uso de substâncias psicoativas são mais frequentes em homens. ${ }^{(9,13)}$

Alguns estudos procuraram demonstrar a correlação entre a etnia e a presença de determinados transtornos mentais,contudo, verificaram que tais correlações eram fracas e estavam sob influência de outros fatores como os ambientais e genéticos. ${ }^{(14)}$ Assim, mesmo com o resultado de $79,9 \%$ de brancos, não foi possível afirmar que esta variável tem correlação com a presença de determinado transtorno mental.

A procedência de 83,3\% dos participantes de Curitiba ou Região metropolitana deve-se àpolítica de descentralização e regionalização dos serviços de saúde promovida pelo Sistema Único de Saúde (SUS), que assegura o acesso aos serviços de saúde, em todos os níveis de atenção, o mais próximo possível da moradia dos usuários. O resultado sobre o modo de moradia, 1,1\%de paciente institucionalizado e 88,3\% vivendo com familiares,é o reflexo da aceleração das ações promovida pelas políticas públicas de saúde mental, como a desinstitucionalização, que buscam dar suporte psicossocial aos pacientes e seus familiares com a finalidade de que estes retornem ao ambiente familiar e à sociedade. ${ }^{(15)}$

Referente à situação conjugal,47,5\% eramcasados e 29,7\% solteiros. A literaturarefere menor prevalência de transtornos mentais em pessoas solteiras em relação às casadas, mas, todavia, ressalta-se que esta associação é fraca e pouco significativa.O nível baixo de satisfação conjugal tem sido correlacionado com o surgimento de transtornos mentais, principalmente a depressão e a ansiedade. ${ }^{(16-17)}$ 
Quase a totalidade dos participantes referiu ter religião. A prática de uma religião é habitualmente um fator de proteção contra o desenvolvimento de transtorno mental, principalmente, depressão, ansiedade e abuso de substâncias, e também pode estar associada à melhor qualidade de vida. Contudo, a prática religiosa pode ser desencadeante de agravos psíquicos quando na presença de rígida disciplina comportamental, em que indivíduos emsofrimento psicológico podem desenvolver estratégias de enfrentamento pouco adaptativas. ${ }^{(16,18)}$

O resultado contido na Tabela 1 referente às variáveis sociais(instrução, ocupação e renda familiar) corrobora a literatura, que as classificam como indicativas para maior risco de surgimento de transtornos mentais nas populações com baixa renda, baixa escolaridade, com instabilidade do vínculo de trabalho (desempregados) e de trabalhadores informais (do lar, autônomo). ${ }^{(16)}$ Todavia, é importante não relacionar diretamente a vulnerabilidade social e a presença de transtornos mentais, pois a variabilidade de sofrimento psíquico, de modo geral, está presente em todas as classes sociais, não cabendo aqui correlacionar "pobreza" com a presença de transtornos mentais. ${ }^{(19)}$

O perfil clínico demonstrou que a unidade de neurologia teve o maior número de casos (n=49), o que se justifica devido às doenças neurológicas, em especial a epilepsia, teremcerto potencial em apresentar complicações psiquiátricas, principalmente transtornos depressivos, ansiedade e sintomas psicóticos. $(16,20)$

Observou-se na Tabela 3que os transtornos depressivos e de ansiedadeforam os mais prevalentes entre as mulheres. Por outro lado, os TMCDU substâncias psicoativas e os TMCDU álcool (10,1\%) tiveram frequências maiores às dos homens, coadunando com a literatura, que demonstra a presença dessa correlação. ${ }^{(4,13,16)}$

A presença de transtornos de ansiedadeé comumente encontrada em populações internadas em hospitais gerais para tratamento de doençasclínicas e pode surgir em resposta temporária à adaptaçãoà hospitalização (transtorno de ajustamento) e como consequência do estresse relacionado com o processo de adoecimento e o tratamento clínico. ${ }^{(4,16)}$

A constatação de que $67 \%$ dos participantes estavam em tratamento psiquiátrico é importante pelo aumento na chance de melhora na qualidade de vida e no prognóstico da doença. Contudo, existe uma preocupação com os 33\% que não estavam, haja vista que a não adesão aos tratamentos pelas pessoas com transtorno mental pode ser consideradaum sério problema na atenção à saúde mental, com consequência como o descontrole dos sintomas, o aumento do número e do tempo de internações psiquiátricas integrais e o aumento no custo dos cuidados de saúde ${ }^{(21)}$.

Obaixo percentual de pacientes em tratamento em Centro de Atenção Psicossocial (CAPS) (3,3\%) contrasta com a ênfase que Política Nacional de Saúde Mental tem dado a este serviço, que é referência nacional para a atenção à saúde mental e dispositivo estratégico da rede de atenção psicossocial, em substituição aos hospitais psiquiátricos, ícones histórico-sociais do modelo manicomial. ${ }^{(15)}$

A presença de comorbidade psiquiátrica em 22,3\% dos participantes converge com a literatura que refere que quase $50 \%$ dos pacientes com transtorno mental acabam por apresentar mais de um diagnóstico psiquiátrico concomitantemente. A complexidade das comorbidades psiquiátricas exige maior qualificação dos profissionais para o reconhecimento de sinais, sintomas, necessidades de cuidados e para a planificação da assistência multiprofissional dentro do contexto psicossocial, tornando-se um desafio para a equipe de hospital geral. (4,8,16) $^{(1)}$

O resultado sobre pensamento suicida entre os participantes teve uma prevalência considerável(50,3\%) e reforça a associação demonstrada na literatura entre transtorno mentale comportamento suicida, principalmente os transtornos do humor, considerados fator de risco para o suicídio. ${ }^{(16)}$ A equipe de enfermagem é a que dispensa maior tempo junto ao paciente e este resultado torna-se extremamente importante para a prática daEnfermagem, na identificação dos sinais característicos do comportamento suicida, bem como na implementação de ações preventivas ao ato suicida nos serviços de saúde. ${ }^{(4,16,22)}$ 
O fenômeno do uso e da dependência de drogas psicoativas torna-se mais complexo quando dados epidemiológicos demonstram que a iniciação do consumo da droga tem sido cada vez mais precoce (crianças e adolescentes), o aumento de doses e o surgimento de drogas com capacidade maior de provocar dependência e também quanto à presença de policonsumo de drogas. Quando se compara a distribuição do uso das drogas e foca-se nos índices do crack percebe-se que sua prevalência é rara $(0,1 \%)$. Contudo, a preocupação das entidades de saúde, baseada em estimativas, é o acelerado aumento destes índices, alto grau de dependência que esta droga tem, além dos efeitos devastadores em curto prazo. ${ }^{(15,16)}$

A dependência do crack constitui um dos mais importantes problemas de saúde pública mundial, haja vista a magnitude dos agravos produzidos por esta droga aos indivíduos, às suas famílias e à sociedade em geral. Entre as diversas implicações no contexto psicossocial, decorrentes da dependência do crack, pode-se destacar: uso cada vez mais cedo, abrangendo grupo de crianças e adolescentes, consequências físicas em curto prazo (cardiopatias, pneumopatias, encefalopatias, doenças sexualmente transmissiveis); danos psíquicos esociais. ${ }^{(23)}$

A partir da consideração das inúmeras consequências físicas causadas pelo crack, pôde-se justificar a identificação de 10,6\% de pacientes dependentes do crack entre os participantes deste estudo que se encontravam hospitalizados para tratamento clínico, e também por este índice estar acima em comparação à prevalência na população em geral.

\section{CONCLUSÃO}

A distribuição de algumas variáveis referentes ao perfil sociodemográfico do paciente se assemelha com dados gerais da população e reforça a afirmação da Organização Mundial de Saúde em que os transtornos mentais podem ocorrer em qualquer fase da vida, não distinguindo sexo, idade, classe social, etnia, mas que alguns grupos são mais suscetíveis: baixa renda, baixa escolaridade, idosos, portadores de doenças crônicas, entre outros.

As características clínicas dos participantes permitiram constatar que as modificações estruturais promovidas pela Atenção à Saúde Mental no Brasil têm avançado no sentido de dar abrangência e maior acessibilidade aos serviços nesta área. Contudo, um dado importante foi o baixo número de casos em tratamento/acompanhamento no CAPS, que é considerado o principal serviço de saúde mental no contexto psicossocial.

Os índices apresentados sobre ideação e tentativa de suicídio servem como subsídio para novos estudos sobre comportamento suicida em hospitais gerais, bem como para criação de estratégias de qualificação dos profissionais de saúde, a fim dediminuir os riscos a este agravo identificado pelos diagnósticos de enfermagem.

As limitações evidenciadas por parte deste estudo foi ter o desenho metodológico transversal, pelo qual não se teve a pretensão de se estabelecer uma relação causal entreos fatores estudados.

Destaca-se a contribuição do conhecimento do perfil, apresentados pelos pacientes em hospitais gerais, para a elaboração de plano de cuidados específicos com suporte aos aspectos psicossociais e físicos. Recomenda-se o desenvolvimento de estudos que correlacionem a prevalência dos transtornos mentaiscom hospitais gerais e com a implementação do Processo de Enfermagem nos serviços de saúde mental. 


\section{REFERÊNCIAS}

1.Coutinho LMS, Matijasevich A, Scazufca M, Menezes PR. Prevalência de transtornos mentais comuns e contexto social: análise multinível do São Paulo Ageing\& Health Study(SPAH). Cad.SaúdePública. [Internet] 2014;30(9) [acessoem 20 ago 2017]. Disponível: http://dx.doi.org/10.1590/0102-311X00175313.

2.World Health Organization (WHO). Depression is a common illness and people suffering from depression need support and treatment. [Internet] Genebra: WHO; 2012 [acesso em 20 ago 2017]. Disponível: http://www.who.int/ mediacentre/news/notes/2012/mental_health_day_20121009/en/.

3.Moll MF, Silva LD, Magalhaes FHL, Ventura CAA. Profissionais de enfermagem e a internação psiquiátrica em hospital geral: percepções e capacitação profissional. CogitareEnferm. [Internet] 2017;(22)2 [acesso em 21 ago 2017]. Disponível: http://dx.doi.org/10.5380/ce.v22i2.49933.

4.Botega NJ, organizador. Prática psiquiátrica no hospital geral: interconsulta e emergência. $4^{a}$ ed. Porto Alegre: Artmed; 2017.

5.Fernandes MA, Pereira RMF, Leal MSM, de Sales JMF, Soares e Silva J. Nursingcaretopsychiatricpatients in a general hospital emergency. RevEnferm UFPI [Internet]. 2016;5(2) [acesso em 22 ago 2017]. Disponível: http://ojs.ufpi.br/index. php/reufpi/article/view/5241/pdf.

6. do Prado MF, Sá MC, Miranda L. O paciente com transtorno mental grave no hospital geral: uma revisão bibliográfica. Saude debate. [Internet]2015;39 [acessoem 25 ago 2017]. Disponível: http://dx.doi.org/10.5935/01031104.2015S005419.

7.Hildebrandt LM, Marcolan JF. Conceptions of nursing staff about psychiatric care in general hospital. Rev Rene. [Internet] 2016;17(3) [acessoem 28 ago 2017]. Disponível:http://dx.doi.org/10.15253/2175-6783.2016000300011.

8. Paes MR, Maftum MA. Difficulties of nursing team of a general hospital in the care of patient with mental disorder. J Nurs UFPE on line. [Internet] 2013;7(9) [acessoem 27 ago 2017].Disponível:https://periodicos.ufpe.br/revistas/ revistaenfermagem/article/view/13675.

9.Brunoni AR. Transtornosmentais comuns na prática clínica. Rev med.[Internet] 2008;87(4) [acesso em 18 dez 2017]. Disponível:http://www.revistas.usp.br/revistadc/article/view/59087/62073.

10.Soeiro RE, Colombo ES, Ferreira MHF, Guimarães PSA, Botega NJ, Dalgalarrondo P. Religião e transtornos mentais em pacientes internados em um hospital geral universitário. Cad. Saúde Pública. [Internet] 2008;24(4) [acesso em 18 dez 2017]. Disponível: http://dx.doi.org/10.1590/S0102-311X2008000400009.

11.Castro-Camacho L, Escobar JM, Sáenz-Moncaleano C, Delgado-Barrera L, Aparicio-Turbay S, Molano JC, et al. Salud mental enel hospital general: resultados delCuestionario de Saluddel Paciente (PHQ) encuatroservicios de atención. Rev. Colomb. Psiquiat. [Internet] 2012;41(1) [acesso em 26 ago 2017]. Disponível: http://dx.doi.org/10.1016/S00347450(14)60069-X.

12.Fonseca CAM. Prevalencia y tendencia de losprincipalestrastornosmentalesenlaCiudad de México; análisis de datosdel 2004 al 2009. Actualidadclin Psicol. [Internet] 2010;(9) [acesso em 23 ago 2017]. Disponível: https:// actualidadclinica.wordpress.com/2010/09/21/prevalencia-y-tendencia-de-los-principales-trastornos-mentales-en-laciudad-de-mexico-analisis-de-datos-del-2004-al-2009/.

13.Eaton NR, Keyes KM, Krueger RF, Balsis S, Skodol AE, Markon KE et al. An Invariant Dimensional Liability Model of Gender Differences in Mental Disorder Prevalence: Evidence from a National Sample. J AbnormSocPsychol. [Internet] 2012;121(1) [acesso em 18 dez 2017]. Disponível: https://www.apa.org/pubs/journals/releases/abn-121-1-282.pdf.

14.Gonzalez JM, Alegría M, Prihoda TJ, Copeland LA, Zeber JE. How the relationship of attitudes toward mental health treatment and service use differs by age, gender, ethnicity/ race and education. SocPsychiatryPsychiatrEpidemiol. [Internet] 2011;46(1) [acesso em 18 dez 2017]. Disponível: http://dx.doi.org/10.1007/s00127-009-0168-4. 
15.Ministério da Saúde (BR). Secretaria de Atenção à Saúde. Departamento de Ações Programáticas Estratégicas. Coordenação Geral de Saúde Mental, Álcool e Outras Drogas. Saúde Mental em Dado. [Internet] Brasília: Ministério da Saúde; 2015 [acesso em 18 dez 2017]. Disponível: http://www.mhinnovation.net/sites/default/files/downloads/ innovation/reports/Report_12-edicao-do-Saude-Mental-em-Dados.pdf.

16.Sadock BJ, Sadock VA. Compêndio de Psiquiatria. 9a ed. Porto Alegre: Artmed; 2007.

17.South SC, Krueger RF, lacono WG. Understanding general and specific connections between psychopathology and marital distress: a model based approach. J Abnorm Psychol. [Internet] 2011;120(4) [acessoem 26 ago 2017]. Disponivel:http://dx.doi.org/10.1037/a0025417

18.Weber SR, Pargament KI. The role of religion and spirituality in mental health. Curropinpsychiatry. [Internet] 2014;27(5) [acesso 28 ago 2017]. Disponível: http://dx.doi.org/10.1097/YCO.0000000000000080.

19. da Gama CAP, Campos RTO, Ferrer AL. Saúde Mental e Vulnerabilidade Social: a direção do tratamento. Rev. latinoam.psicopatol. fundam. [Internet]2014;17(1) [acesso 27 ago 2017]. Disponível:http://dx.doi.org/10.1590/S141547142014000100006 .

20. Terra JR, Valerio NI, de Oliveira GNM. Correlações clínicas entre as epilepsias e transtornospsiquiátricos: considerações da literatura. J. epilepsyclinneurophysiol. [Internet]2013;19(1) [acesso em 18 dez 2017]. Disponível: http://files.bvs.br/upload/S/1676-2649/2013/v19n1/a4875.pdf.

21. de Sousa LPCS, Vedana KGG, Miasso AI. Compliance with medication treatment by people with anxiety disorder. CogitareEnferm. [Internet] 2016;21(1) [acesso 28 ago 2017].Disponível: http://dx.doi.org/10.5380/ce.v21i1.43510.

22.Simon H. Prise en charge infirmière du suicidant. Soins. [Internet] 2017;62(814) [acesso em 18 dez 2017]. Disponível:http://dx.doi.org/10.1016/j.soin.2017.02.010.

23. Horta RL, Schäfer JL, Coelho LRM, Rodrigues VS, de Oliveira MS, Teixeira VA. Condições associadas a prejuízo de desempenho em habilidades sociais em uma amostra de conveniência de usuários de crack. Cad. Saúde Pública [Internet] 2016;32(4) [acesso em 18 dez 2017]. Disponível:http://dx.doi.org/10.1590/0102-311X00010715. 